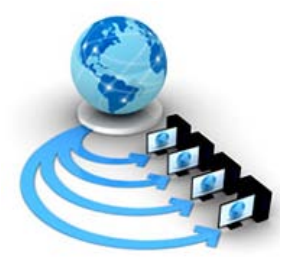

International Journal of Advanced Research in Computer Science

SURVEY REPORT

\title{
SURVEY ON PREDICTING SLE IN PREGNANT WOMEN BY USING CLASSIFICATION ALGORITHM
}

\author{
Dithy MD \\ Research Scholar \\ Department of Computer Science \\ Sri Ramakrishna College of Arts and science \\ for Women, Coimbatore, India
}

\author{
Dr. V. Krishnapriya \\ Head of the department \\ Department of Computer Science \\ Sri Ramakrishna College of Arts and science \\ for Women, Coimbatore, India
}

\begin{abstract}
Systemic lupus erythematosus (SLE) is a chronic immune disease that affects both male and female, but mostly it is caused by the female during menstrual periods which almost affect all the organs of the human body. Predicting this disease is difficult in the early stage the patients suffering from SLE live their life with treatment. Women are having SLE faces many risks during pregnancy due this abortion occur and infant is born with less weight. After predicting the patient's disease, it is difficult to store in a huge database, it includes patient's complete history. Thus the data mining, classification approach is used for the competent analysis of the information to predict the disease in advance. This review is about predicting the database of the patients having SLE by using classification algorithm.
\end{abstract}

Keywords: SLE, data mining, decision tree, artificial neural network

\section{INTRODUCTION}

Systemic lupus Erythematosus (SLE) is a chronic autoimmune disease with a worldwide distribution. SLE, as an inflammatory multi-system disease with unknown etiology, has different clinical manifestations and symptoms, laboratory signs, and prognosis in patients [1]. It affects all parts of the body like skin, joints, kidneys, lungs, nervous system and other organs of the body the disease is mild, moderate and severe disease which can vary from mild to death of fetal. Pregnancy in SLE women is a major challenge Woman suffering from SLE who want to become pregnant face risk during pregnancy and baby is born with unhealthy [2]. Women affected by SLE lead by fetal loss in the form of abortion before 20 weeks of growth or stillbirth after 20 weeks of growth. In 1960s 43\% of fetal loss has been decreased and in 2000s 17\% has decreased in SLEaffected women [3]. Although in recent years fetal survival has increased due to better understanding and causes of abortion in SLE patients fetal loss continues to be higher among SLE-affected women [4]. In the UK, the highest incidence is in Black Caribbean women (31.5/100,000 person-years), and The incidence in Black other $(22.3 / 100,000)$ and Black African $(13.8 / 100,000)$ women is also increased. The rates in Pakistani $(10.0 / 100,000)$, Indian (9.9/100,000), and Chinese women $(9.4 / 100,000)$ are lower with the lowest incidence in White women $(6.7 / 100,000)$ [5]. The stages of the disease are mild Medical data mining is large in numbers and it's difficult to store and maintain. A variety of algorithms such as neural networks, fuzzy logic, regression, Bayesian networks algorithms have been applied in different fields of healthcare. By using Data mining technique, it can efficiently predict the data easily.

\section{LITERATURE REVIEW}

Srinivas [6] analyzed a massive volume of health care data using rule based, decision tree and Naïve Bayes classification method. The heart attacks need an effective and efficient algorithm like naïve creedal classifier, rule set, classifier (C4.5) and One Dependency Augmented Naïve Bayes classifier.

George Bertisias et.al. [7] Explained the key events of pathogenesis of SLE and analyzed the genetics, epigenetic, hormonal and environmental factors found in the disease. The author suggested the classification criteria of lupus and limitations for diagnostic purposes. The clinical manifestation of SLE in the musculoskeletal, renal, central nervous system, dermatological, respiratory, hematological systems and gastrointestinal were also described

hadijeh Paydar et.al. [8]. Used CDSS by developed, physicians in predicting the outcome of pregnancy in SLEaffected women and evaluated a CDSS based on an artificial neural network (ANN) application.

Ceccarelli et.al. [9]. Explained The use of the longitudinal data from the Sapienza Lupus Cohort, including laboratory and experimental substance resulted capable to assemble a statistical representation, potentially identifying patients at risk to develop damage by applying RNNs in predicting chronic damage.

P. Lapuerta et.al. [10]. Explained there are no other studies related to developing models using neural networks or other machine-learning algorithms for predicting the pregnancy outcomes of SLE-affected women, researchers successfully applied neural networks for either multivariate analysis or predicting a range of diseases or for disease risk estimation.

Kurt et al., [11]. Compared the performance of classification methods in order to predict the presence of coronary artery disease (CAD) and found that those areas 
under the ROC curves were $0.783,0.753,0.745,0.721$, and 0.675 for MLP, logistic regression (LR), classification and regression tree (CART), and RBF, respectively .

\section{OVERVIEW OF DATA MINING}

Data mining is used in various fields such as in business, social media, healthcare because all these fields contains a huge amount of data which cannot be able to store and retrieve by using data mining, classification algorithm store and retrieval process can be done many researchers have proved it in the fields of computer science. Data mining is defined as extracting the hidden pattern of information from huge database; it is also called as a knowledge discovery process (KDD). Three steps involved are in dataming are Exploration, Pattern identification, Deployment. Classification is one of the dataming techniques which develop a pre-classified data to develop a model from large databases (i.e.) used in fraud detection and credit card analysis neural network-based classification algorithms are suited for this approach. Clustering is identification of similar classes of objects. Association rule is used to find frequent item set among large data sets.

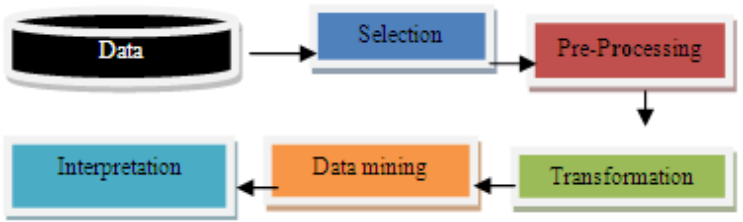

Figure 1. Knowledge discovery process (KDD).

\section{SLE IN PREGNANT WOMEN USING DATA MINING}

Khadijeh Paydar et.al [12] Pregnancy among systemic lupus erythematosus (SLE) affected women is highly associated with poor outcomes. Datasets of SLE patients were collected from Charity Hospital retrospective analysis of 149 pregnant women with SLE dataset was taken in that who (104 pregnancies) and a specialized clinic (453 pregnancies) from the year 1982 to 2014 by using a binary logistic regression preprocessing was done. Artificial neural networks were applied to predict the pregnancy outcome. Confusion matrix was used to evaluate and then select the most effective network. MATLAB was applied to design the neural networks. 45 potential variables were analyzed. The accuracy $(90.9 \%)$, sensitivity (80.0\%), and specificity (94.1\%) of the test data for the MLP network were achieved. Therefore, the MLP network was selected as the most accurate network for prediction of pregnancy outcome. Factors such as high blood pressure, kidney failure, heart attack, anemia, abortion are the symptoms of SLE.

\section{CONCLUSION}

The present study aimed at predicting SLE in pregnant women by using a classification algorithm and storage, retrieval process was done. In future, by using benchmark datasets regarding anemia in pregnant women can be taken from different repositories, storage and retrieval process can be done and by applying different classification algorithm. Anemia is one of the symptoms of SLE in pregnant women.

\section{REFERENCES}

[1] M. Akbarian, S.T. Faezi, F. Gharibdoost, F. Shahram, A. Nadji, A.R. Jamshidi, M. Akhlaghi, N.Shafaie, M. Akhlaghkhah, F. Davatchi, Systemic lupus erythematosus in Iran: a study of 2280 patients over 33 years, Int. J. Rheum. Dis. 13 (4) (2010) 374-379.

[2] M.A. Khamashta, Systemic lupus erythematosus and pregnancy, Best Pract. Res. Clin. Rheumatol.20 (4) (2006) 685-94.

[3] M. Akbarian, A. Rostamian. Systematic Lupus Erythmatosus,TehranUniversityof MedicalMScience, Tehran, 2011.

[4] A.G. Singh, V.R. Chowdhary, Pregnancy-related issues in women with systemic lupuserythematosus, Int. J. Rheum. Dis. 18 (2) (2014) 172-81.

[5] C.C. Mok, R.W.S. Wong, Pregnancy in systemic lupus erythematosus, Postgrad. Med. J. 77 (905) (2001) 157-65.

[6] Srinivas, "Novel approach for heart prediction verdict using data mining technique", International Journal of Computer Science and Engineering. 2010 .

[7] George Bertsias, Ricard Cervera, Dimitrios Boumpas, "Systemic Lupus Erythematosus: Pathogenesis and Clinical features”, EULAR textbook on Rheumatic Disease.

[8] Khadijeh Paydar, Sharareh R. Niakan Kalhori, Mahmoud Akbarian, Abbas Sheikhtaheri, "A Clinical Decision Support System for Prediction of Pregnancy Outcome in Pregnant Women with Systemic Lupus Erythematosus.

[9] Ceccarelli, Sciandrone,M, Perricone, Galvan G, Morelli F, Vicente LN, "Prediction of chronic damage in systemic lupus erythematosus by using machine-learning models”. . 2017 March.

[10] P. Lapuerta, S.P. Azen, L. LaBree, Use of neural networks in predicting the risk of coronary Artery disease, Comput. Biomed. Res. 28 (1) (195) 38-52.

[11] I. Kurt, M. Tour, A.T. Quorum, Comparing the performances of logistic regression, classification and Regression tree, and neural networks for predicting coronary artery disease, Expert Syst. App. 34 (1) (2008) 366-74.

[12] Khadijeh Paydar, Sharareh R. Niakan Kalhori, Mahmoud Akbarian, Abbas Sheikhtaheri, "A Clinical Decision Support System for Prediction of Pregnancy Outcome in Pregnant Women with Systemic Lupus Erythematosus. 